\title{
Optimization of Automatic Power Monitoring and Control Method in HVDC Projects
}

\author{
Yanhong Shen, Rui Zhang, Haoran Huang, Guohui Rao \\ HVDC dept. XJ Group Co. Ltd, Xuchang, Henan, 461000, China
}

\begin{abstract}
The construction of UHVDC (ultra high voltage direct current) transmission integrates the regional power grids into a whole grid in China, which increase the importance of power grid stability. Automatic power control helps operation personnel reduce the burden of work, but it may also take the power control error risk. The paper briefly investigates an improved method to reduce manual input and the burden of monitoring work for automatic power control. The improved method is based on the Yunnan-Guangdong II 800Kv UHVDC transmission project station SCADA (supervisory control and data acquisition) system. It does well in the project performance and reduce the operator's work burden effectively.
\end{abstract}

\section{Introduction}

The high voltage direct current transmission power is at present an important way to solve the problem of high voltage, large capacities, long-distance power transmission and power gird interconnection in the world [1-3]. Due to the characteristics of energy and load demand in our country the reverse distribution, HVDC transmission becomes the hotspot of electric power construction. Both the back-to-back and long-distance HVDC transmission project connects two AC large power grids through the HVDC. Especially when one end is weak grid while another end is strong grid, stability is the highest priorities. The slightest mistake may appear large area blackout, such as America blackout and the London blackout [4-5].

All the HVDC projects in China are designed according to someone on duty mode [6]. The operation personnel input the power reference value at the appointed time according to the dispatching instruction and calculate the power fluctuation rate manually. They do the repetitive work every day. Even with automatic power control function, the operation personnel also need to input value and to calculate rate and must to focused attention to the power transmission state. If there is a problem and it is not handled in a timely manner, it may bring serious consequences. However, the converter operation personnel are not only responsible for monitoring the screen, but also other related chores. How to carry on automated processing to reduce the probability of human error will help for the stable of HVDC transmission system. In the paper improved method is proposed to reduce the input work and make monitoring work easily.

\section{Automatic power control Principle}

The power control mode is one of the key parameters to HVDC power transmission characters. It is divided basically into two categories: power mode and current mode [7]. Power mode is a kind of control function of pole control level. Usually in the normal operation, control system keeps the system power to the setting value by operation personnel. The structure diagram of the automatic power control plan system is shown in Figure 1.

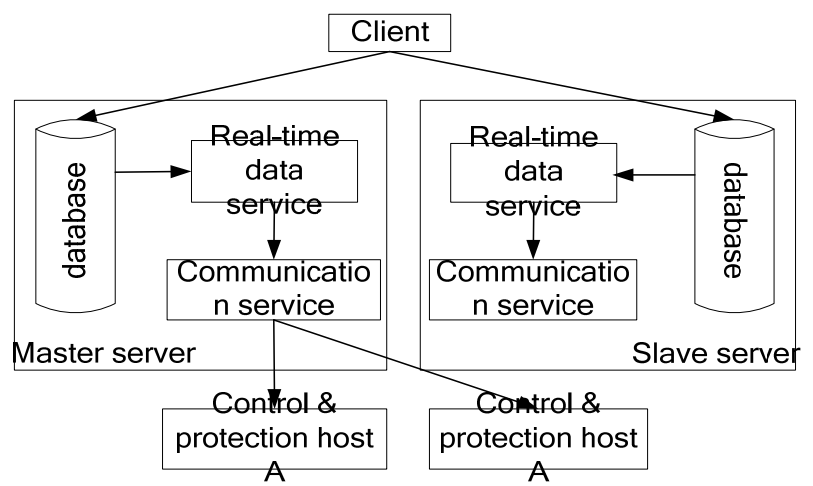

Figure 1. The automatic power control plan system diagram Note: the communication service in the slave server does not send the plan data to the control and protection host $\mathrm{A}$ and $\mathrm{B}$.

At present the automatic power control at home and abroad are implemented in the SCADA system. Generally it needs to run manually starting time and slope calculation. That not only increases work burden, but also is wrong easily. Not only the control and protection system but also the SCADA system must have redundancy measures in HVDC transmission system. The operation personnel input the automatic power plan value through the client interface. Then automatic power plan data is stored in two mutual standby server databases. The master server real-time processing procedure gets the plan data from the database and sends the plan data to the redundant control system through the master server communication program.

The scheduled value file is sent to converter station through telecontrol communication by the dispatch center. From the time 0 hour, there is a power value every 15 minutes in the file. As shown in figure 2, point A, B, C, D, etc are plan values which are on the red line. The red line is the running results what the dispatcher wants. The black line is the practical operation results. $\Delta t$ is time period which the Bipolar power reached ahead. $\Delta \mathrm{t}$ is used to deal with the evaluation which the dispatch to the operation personnel. Since in practice, there may be some delay in the filed of converter station. At the same time, not all the power values will be gotten from the 
dispatch's file. Only the values in turning points are necessary.

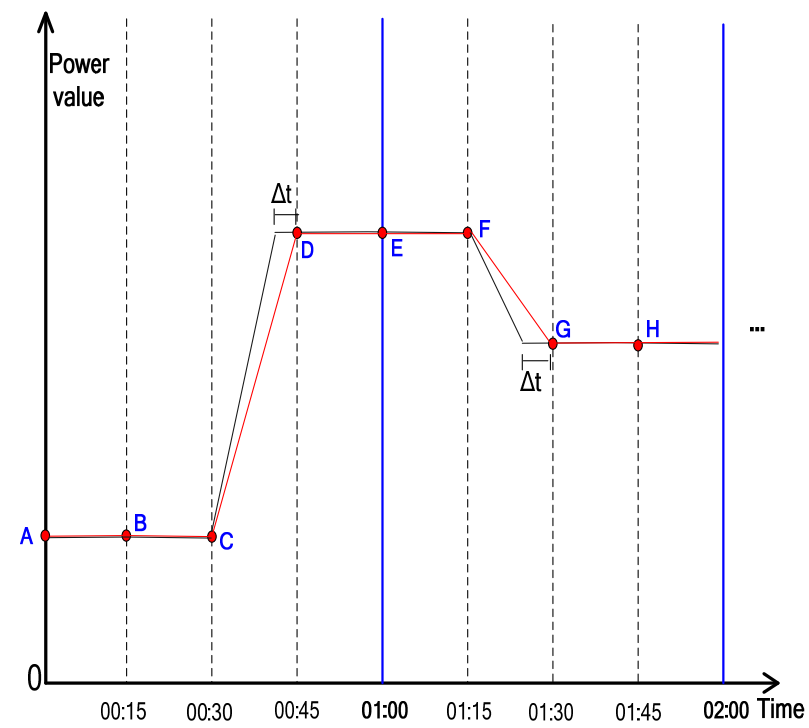

Figure 2. The automatic power curve diagram

According to the characteristics of the dispatch file that one power value every 15 minutes, SCADA issues the reference value every 15 minutes under the automatic power control mode.

\section{Principle Optimization Implementation}

and

In the paper, combined with the Yunnan-Guangdong UHVDC transmission project, the improved method is introduced in detail in the following .

In the design of converter station, the SCADA and RCI (remote control interface) are in the same LAN. After the dispatcher sends the scheduling power value to the station, it can be detached by the RCI service. The operation personnel who have the edit access only need to login the automatic power curve page. If there have a new schedule file, the HMI (human machine interface) client will get it and load it. The operation personnel only need to carefully check the start time and the end time, then confirmed it and upload the automatic power curve data to the database. The automatic power curve data is checked actively is shown in Figure 3.

The power slope value is calculated according to the formula [power difference $\times 60 /$ time (second)], where the power difference is the difference between the present power and the previous power, for example the power difference is $1000 \mathrm{MW}-850 \mathrm{MW}=150 \mathrm{MW}$, the time difference is $15 \times 60-\Delta \mathrm{t}$ (second), namely $900-\Delta \mathrm{t}$, If $\Delta$ $\mathrm{t}=0$, the slope value is $150 \times 60 / 900=10 \mathrm{MW} / \mathrm{Min}$; If $\Delta$ $\mathrm{t}=20(\mathrm{~s})$, the slope value is $150 \times 60 / 880=10.23 \mathrm{MW} / \mathrm{Min}$, according to the principle of 1 rounded, slope value is $11 \mathrm{MW} / \mathrm{Min}$. In order to make the power change smoothly, the slope of each time interval is minimum value and it may be not the same. That ensures the power regulation can be successfully completed.

When the automatic power curve is running, the operation personnel need to focus on the sequence event record. The bipolar power value will only appear in the diagram, not in the sequence event record, which may be made mistakes by negligence.

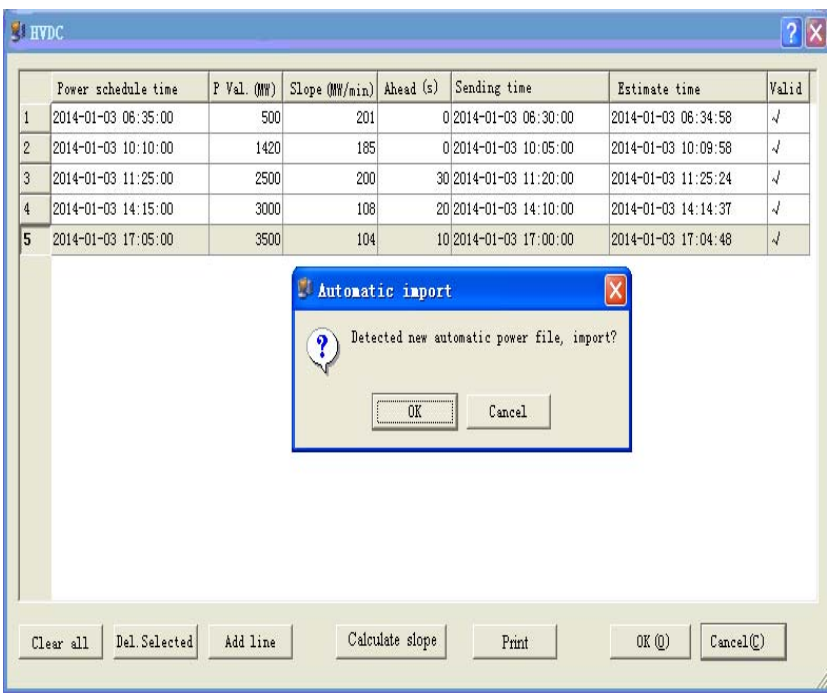

Figure 3. The automatic power curve checked diagram

When the automatic power curve is running, it can prevent the unexpected thing through comparing the measured value. As shown in figure 4, the right side shows the three lines labels.

Red curve is drawn according to the dispatch schedule values. The yellow curve is drawn based on the measurement of bipolar power reference value and the blue curve is drawn based on the measurement of bipolar power measurement value. It can be gotten the state whether the normal operation of the automatic power curve through the deviation between red curve and blue curve. Of course, it may be the deviation between yellow curve and blue curve. At the same time, the corresponding sequence event record will be shown caused by the deviation. Because the event is configured with the highest level, its appearance makes the alarm sounds. So that ensures the operation personnel to pay attention to it. The deviation threshold value can be set through the setting dialog. When the deviation exceeds the threshold, there will have event appear in the sequence event record. On the contrary case there will not have event appear. The frequency of occurrence and event trigger are set through the setting dialog. The monitor curves and setting dialog as shown in figure 4.

A perfect remainder mechanism guarantees the automatic power curve works well. When the power mode from manual to automatic, not only an event which description is "automatic power mode starting" appears, but also another event appears which shows how many seconds later the power will be adjusted and how much the corresponding slope is. When the first power point is adjusted according to the setting, an event will be used to show the next power point information, and so on. That helps the operation personnel master the state of the power turning point and prevent command leakage. 


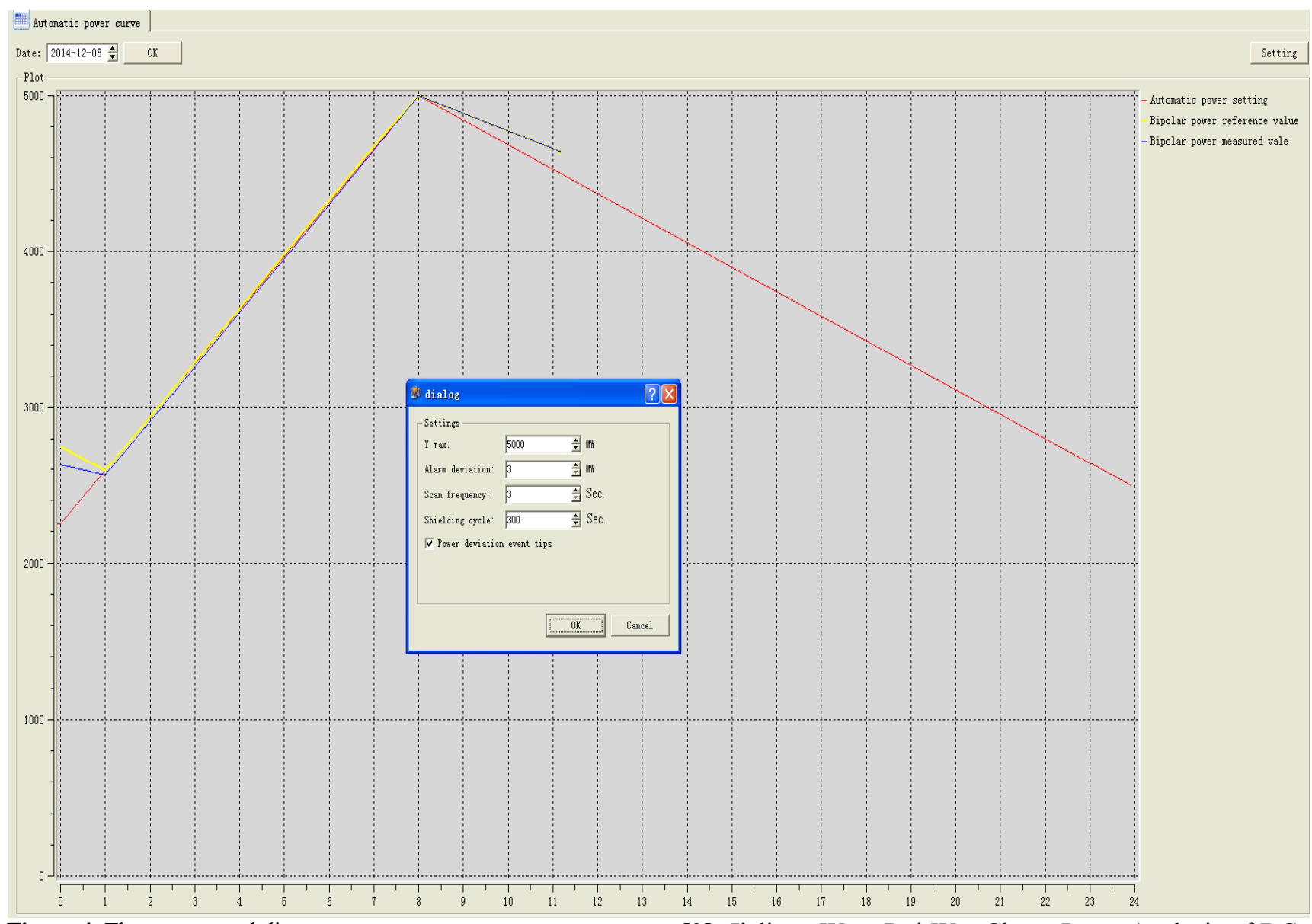

Figure 4. The system tool diagram

\section{Application in Projects}

The improved system has been applied in the Yunnan-Guangdong II $800 \mathrm{Kv}$ UHVDC project, all the function is working normally and has made good results.

\section{Summary}

Based on the analysis of automatic power control system, combined with the engineering practice, the automatic power curve was optimized. It is improved the automatic power curve function. At the same time, it convenient the operation personnel monitor and control. The practice shows that the optimization is successful. It can fully meet the demand of the operation personnel. The application of the system improves the working efficiency of the operation personnel and reduces the probability of the accident [8].

\section{References}

[1] Xiaoli Li, Zhusheng Chen. Summary of Ultrahigh Voltage Direct Current Transmission Technology. guangxi eletric power press.1, p.23-26. (2009)

[2] Wanjun Zhao. High Voltage Direct Current transmission Engineering Technology. Chinese Power Press. (2004)
[3] Jialiang Wen, Rui Wu, Chang Peng. Analysis of DC Grid Prospects in China. Proceedings of the CSEE. Vol 32 (13). p. 7-11, (2012)

[4] Deqiang Gan, Jiangyi $\mathrm{Hu}$ and Zhenxiang Han. Thought on the several blackouts of 2003 in the world. Automation of Electric Power Systems. vol 28, pp. 1-4,9. (2004)

[5] Baosheng Tang. Blackout in South of London and Its Lessons. Power System Technology. vol 27 (11), pp 1-5,12. November (2003).

[6] Shiping E, Haoran Huang. Research and Implementation of Automatic Power Control for HVDC. 5, p. 1-3, (2013)

[7] Hongtao Wang, Runhua He and Pengcheng Shi. The Power Transmission Mode and Their Exchange Conditions of Tianshengqiao-Guangzhou HVDC Project. Southern Power System Technology. vol 6 (1), pp. 30-33. (2012)

[8] Zhenya Liu. Ultra-High voltage AC \& DC Grid. China electric power press. 12, p. 16-17. (2013) 